\title{
AEGIS20: A RADIO SURVEY OF THE EXTENDED GROTH STRIP
}

\author{
R. J. Ivison, ${ }^{1}$ S. C. Chapman, ${ }^{2}$ S. M. Faber, ${ }^{3}$ Ian Smail ${ }^{4}$ A. D. Biggs, ${ }^{1}$ C. J. Conselice, ${ }^{5}$ \\ G. Wilson, ${ }^{6}$ S. Salim, ${ }^{7}$ J.-S. Huang ${ }^{8}{ }^{\text {and S. P. Willner }}{ }^{8}$ \\ Received 2006 May 14; accepted 2006 October 2; published 2007 April 5
}

\begin{abstract}
We describe AEGIS20 - a radio survey of the Extended Groth Strip (EGS) conducted with the Very Large Array (VLA) at $1.4 \mathrm{GHz}$. The resulting catalog contains 1123 emitters and is sensitive to ultraluminous $\left(10^{12} L_{\odot}\right)$ starbursts to $z \leq 1.3$, well matched to the redshift range of the DEEP2 spectroscopic survey in this region. We use stacking techniques to explore the microjansky-level emission from a variety of galaxy populations selected via conventional criteria-Lyman break galaxies (LBGs), distant red galaxies (DRGs), UV-selected galaxies, and extremely red objects (EROs) - determining their properties as a function of color, magnitude, and redshift and their extinction-free contributions to the history of star formation. We confirm the familiar pattern that the star formation rate (SFR) density, $\rho_{\star}$, increases by at least a factor of $\sim 5$ from $z=0$ to 1 , although we note highly discrepant UV- and radio-based SFR estimates. Our radio-based SFRs become more difficult to interpret at $z>1$ where correcting for contamination by radio-loud active galactic nuclei (AGNs) comes at the price of rejecting luminous starbursts. While stacking radio images is a useful technique, accurate radio-based SFRs for $z \gg 1$ galaxies require precise redshifts and extraordinarily high fidelity radio data to identify and remove accretion-related emission.
\end{abstract}

Subject headings: cosmology: observations - galaxies: evolution — galaxies: formation

Online material: color figure

\section{INTRODUCTION}

The tight correlation between radio and far-IR emission for star-forming galaxies (Helou et al. 1985; Kovács et al. 2006) allows us to push dust-independent surveys down to lower SFRs than is possible in the confusion-limited far-IR/submillimeter wave bands. Moreover, the high mapping speed of facilities such as the Giant Metrewave Radio Telescope means that we can quickly obtain the large samples of faint sources needed for reliable analyses.

The bulk of the far-IR background seen by $C O B E$ (Fixsen et al. 1998) most likely arises from a large population of luminous and ultraluminous IR galaxies (LIRGs and ULIRGs, respectively), their energy originating from dust-obscured star formation and accretion. Individually less luminous than submillimeter galaxies, with $L_{\text {bol }} \sim 3 \times 10^{11} L_{\odot}$, these galaxies are believed to be sufficiently numerous to dominate $\rho_{\star}$ at $z \sim 1$ (Dole et al. 2006).

In this Letter we present a new panoramic radio surveyAEGIS20 - undertaken with the NRAO's ${ }^{9}$ VLA as part of the AllWavelength Extended Groth Strip International Survey (AEGIS; Davis et al. 2007). AEGIS20 was tuned to detect ULIRGs robustly at $z \sim 1$, with a noise level of $10 \mu \mathrm{Jy}$ beam $^{-1}$ at $1.4 \mathrm{GHz}$. The resulting catalog, available electronically, contains $\sim 10^{3}$ faint radio

\footnotetext{
${ }^{1}$ UK Astronomy Technology Centre, Edinburgh EH9 3HJ, UK.

${ }^{2}$ Astronomy Department, California Institute of Technology, Pasadena, CA 91125.

${ }^{3}$ Department of Astronomy and Astrophysics, University of California, Santa Cruz, CA 95064.

${ }^{4}$ Institute for Computational Cosmology, Durham University, Durham DH1 3LE, UK.

${ }^{5}$ School of Physics and Astronomy, University of Nottingham, Nottingham NG7 2RD, UK.

${ }^{6}$ Spitzer Science Center, California Institute of Technology, Pasadena, CA 91125.

${ }^{7}$ Department of Physics and Astronomy, University of California, Los Angeles, CA 90095.

${ }^{8}$ Harvard-Smithsonian Center for Astrophysics, Cambridge, MA 02138.

${ }^{9}$ The National Radio Astronomy Observatory is a facility of the NSF operated under cooperative agreement by Associated Universities, Inc.
}

sources - an order of magnitude more than the $5 \mathrm{GHz}$ survey of this region by Fomalont et al. (1991); nearly half are expected to have optical spectra provided by the DEEP2 survey, many with redshifts, as well as photometry across a wide range of wavelengths.

The future goal of AEGIS20 is to measure the $1.4 \mathrm{GHz}$ luminosity function, track the evolution of SFRs in LIRGs and ULIRGs, and, using a measure of the local galaxy density of each radio source, study the history of star formation as a function of environment. Here we present the AEGIS20 catalog and utilize the radio image to estimate SFRs for a number of independent and overlapping galaxy populations selected via conventional criteria.

\section{OBSERVATIONS AND DATA REDUCTION}

Data were obtained at $1.4 \mathrm{GHz}$ during 2003-2005 with the VLA in its B configuration, acquiring seven $3.125 \mathrm{MHz}$ channels every $5 \mathrm{~s}$ at each of four intermediate frequencies. We obtained data in six positions, spaced by $15^{\prime}$ (see Davis et al. 2007), concentrating in the northern half of the EGS because of the proximity of $3 \mathrm{C} 295\left(S_{1.4 \mathrm{GHz}}=23 \mathrm{Jy}\right)$. Around $18 \mathrm{hr}$ of data were acquired for each of the field positions, cycling through them between scans of $1400+621$ and $1419+543$ to monitor bandpass, amplitude, and phase. Absolute flux calibration was set using 3C 286.

Calibrated visibilities and associated weights were used to generate mosaics of $37 \times 512^{2} \times 0.8 \operatorname{arcsec}^{2}$ pixel images to quilt the VLA's primary beam in each EGS field position. CLEAN boxes were placed tightly around all sources, and a series of IMAGR and CALIB tasks were run, clipping the $u v$ data after subtracting CLEAN components generated by the third iteration of IMAGR. The central images from each of the pointings were then knitted together using FLATN, ignoring data beyond the primary beam's half-power point, to produce a large mosaic. The synthesized beam is circular, with a FWHM of $\sim 3.8^{\prime \prime}$. 


\section{SAMPLE DEFINITION}

To define a sample of radio sources, we searched signal-tonoise ratio $(\mathrm{S} / \mathrm{N})$ images using the $\mathrm{SAD}$ detection algorithm, emulating the technique described by Biggs \& Ivison (2006). Sources with $\geq 4 \sigma$ peaks were fitted with two-dimensional Gaussians using JMFIT, and those with $\geq 5 \sigma$ peaks that survived were fitted in total intensity. Sources with sizes equal to or smaller than the restoring beam were considered unresolved. We make no correction for bandwidth smearing in the catalog; this is a small effect $(\sim 5 \%)$ given our mosaicking strategy and the use of the B configuration. We detect 38, 79, 171, 496, and 1123 sources with $S_{1.4 \mathrm{GHz}} \geq 2000, \geq 800, \geq 320, \geq 130$, and $\geq 50 \mu \mathrm{Jy}$, where the $5 \sigma$ detection limits at 130 and $50 \mu \mathrm{Jy}$ cover 0.73 and $0.04 \mathrm{deg}^{2}$, respectively. Confusion is not an issue; the source density on an $\operatorname{arcmin}^{2}$ scale is $<0.01$ beam $^{-1}$.

$S_{1.4 \mathrm{GHz}}=50 \mu \mathrm{Jy}$ corresponds to rest-frame $1.4 \mathrm{GHz}$ luminosities, $L_{1.4 \mathrm{GHz}}$, of $0.44,2.3,6.0$, and $12 \times 10^{23} \mathrm{~W} \mathrm{~Hz}^{-1}$ and SFRs of 50, 275, 725, and $1430 M_{\odot} \mathrm{yr}^{-1}$ at $z=0.5,1.0,1.5$, and 2.0, respectively (for $\Omega_{m}=0.27, \Omega_{\Lambda}=0.73, H_{0}=71 \mathrm{~km}$ $\mathrm{s}^{-1} \mathrm{Mpc}^{-1}$ [Spergel et al. 2003], a Salpeter initial mass function [IMF] with $d N / d M \propto M^{-2.35}$ over $0.1-100 M_{\odot}$, and $S_{\nu} \propto \nu^{-0.8}$ ).

AEGIS20 covers $57 \%$ of the existing DEEP2 region (Davis et al. 2007), with $\sim 7900$ unique redshifts available in the $0.28 \mathrm{deg}^{2}$ common to both surveys. DEEP2 thus covers $35 \%$ of AEGIS20, although its BRI imaging covers $90 \%$ of AEGIS20 (93\% of cataloged sources). Of the AEGIS20 sources with optical imaging, $\sim 36 \%$ have $R_{\mathrm{AB}}<24.1$ counterparts within 1 ". Since the DEEP2 targeting rate is $\sim 70 \%$, the inclusion rate on DEEP2 masks for faint radio emitters is $\sim 25 \%$. At present, $\sim 100$ of the targeted AEGIS20 radio sources have DEEP2 redshifts-a very high success rate.

\section{THE RADIO PROPERTIES OF DISTANT GALAXY POPULATIONS}

The wealth of multifrequency data in AEGIS allows us to mimic the selection of galaxy populations such as DRGs $(J-K>2.3$ and expected to lie at $1.9<z<3.5$; Franx et al. 2003), as well as LBGs (Steidel et al. 2003) and EROs. We investigate the radio properties of several such populations in this section, taking them roughly in order of increasing redshift.

We expect to detect only a small fraction of distant galaxies at radio frequencies. In such situations it is common to assess the emission from a galaxy population using a stacking analysis, accomplished either by extracting and co-adding postage stamps centered on the galaxies of interest ("image stacking") or by co-adding flux densities measured at the positions of the galaxies ("pixel stacking"). We adopt both approaches here. To determine the signal lost by pixel stacking, we employed radio emitters with $\mathrm{S} / \mathrm{N}=5-20 \mathrm{pixel}^{-1}$, finding a difference of only $3.9 \%$ between the values returned at the positions of the emitters and cataloged AEGIS20 flux densities. Monte Carlo simulations show that the mean $S_{1.4 \mathrm{GHz}}$ determined by pixel stacking are slightly skewed $(+0.1 \mu \mathrm{Jy}$, typically) but are otherwise well described by Gaussian statistics; medians are affected at the $<0.01 \mu \mathrm{Jy}$ level. $S_{1.4 \mathrm{GHz}}$ values have been corrected for bandwidth smearing $(+5.0 \%)$ and for pixel-stacking losses $(+3.9 \%)$, and we have excluded galaxies in noisy regions $\left(\sigma_{1.4 \mathrm{GHz}}>30 \mu \mathrm{Jy}\right.$ beam $\left.^{-1}\right)$.

We must excise emission due to accretion if we are to determine accurate radio-based SFRs. Morphological classification of most radio emitters is not feasible at the resolution of our data, spectral indices are not to hand, and the availability and reliability of AGN indicators at shorter wavelengths differs widely across the EGS. Radio-loud AGNs were thus identified and rejected via a $L_{1.4 \mathrm{GHz}}$ limit. Following Condon (1992), we adopt $L_{1.4 \mathrm{GHz}}<10^{24} \mathrm{~W} \mathrm{~Hz}{ }^{-1}$ for normal galaxies, an order of magnitude below the break in morphology and luminosity noted by Fanaroff \& Riley (1974). We quote the noise-weighted mean $S_{1.4 \mathrm{GHz}}$; where AGN contamination is extreme $(>5 \%)$, we quote the median, noting the number of obvious AGNs. One unfortunate consequence of excising radio-loud AGNs on the basis of $L_{1.4 \mathrm{GHz}}$ is the exclusion of distant hyperluminous starbursts lying on the far-IR/radio correlation ( $\$ 5$ ).

We begin with a UV-selected catalog containing 4426 galaxies detected at $230 \mathrm{~nm}$ by $G A L E X$, with DEEP2 redshifts, i.e., $R_{\mathrm{AB}}<24.1$, excluding objects with AGN flags (S. Salim et al. 2007 , in preparation). Of these, 3908 lie within the $0.28 \mathrm{deg}^{2}$ of common areal coverage with AEGIS20. We compare SFRs determined in two ways - via their UV and radio properties, $S_{F R}$ and $\mathrm{SFR}_{\mathrm{rad}}$-for the same galaxies. We use UV-based, extinctioncorrected SFRs, derived by comparing observed spectral energy distributions (SEDs) with those of model galaxies exhibiting a wide range of properties and SF histories (Salim et al. 2005). Appropriate volume corrections for the GALEX-selected sample are difficult to determine due to a complex selection function dependent on UV/optical magnitudes and spectral characteristics. We therefore correct for the rate with which GALEX detects DEEP2 galaxies, which is known to fall from $90 \%$ to $75 \%$ to $60 \%$ at $z=0.2$ to 1.0 to 1.4 , but not for our steadily decreasing sensitivity to low-luminosity galaxies (Arnouts et al. 2005). Because of this, we are limited to discussing the ratio of $\rho_{\star(\mathrm{UV})}$ and $\rho_{\star(\mathrm{rad})}$ within the sample.

Robust radio detections of the UV sample were possible by pixel stacking over $\Delta z=0.2$ bins. Only 11 radio-loud AGNs were identified via $L_{1.4 \mathrm{GHz}}, \ll 1 \%$ of the total; having rejected these, noise-weighted means provide the most appropriate measure of SFR for this sample (Table 1). SFR ${ }_{U V}$ and $\mathrm{SFR}_{\text {rad }}$ per UV-selected galaxy both increase with redshift, unsurprisingly since we are probing more UV-luminous galaxies at larger distances. For $z=0-1, \rho_{\star(\mathrm{UV})}$ remains fairly constant, while $\rho_{\star(\mathrm{rad})}$ rises rapidly. It may seem puzzling that $\rho_{\star(\mathrm{UV})}$ at $z \sim 0$ is an order of magnitude higher than $\rho_{\star(\mathrm{rad})}$ (Fig. 1)- $\rho_{\star(\mathrm{rad})}$ should be sensitive to all recent star formation, obscured and unobscured, for a constant IMF-however, Bell (2003) showed that $L_{\mathrm{far}-\mathrm{IR}} / L_{\mathrm{UV}}$ varies by a factor of $\gtrsim 30$ between $0.01 L_{\star}$ and $3 L_{\star}$ and that radio data underestimate SFRs in low-luminosity galaxies typical of those detected locally by GALEX. Hopkins $\&$ Beacom (2006) argue that for the full picture, we should add $\mathrm{SFR}_{\mathrm{UV}}$ and $\mathrm{SFR}_{\mathrm{rad}} ; \rho_{\star(\mathrm{rad})}$ and $\rho_{\star(\mathrm{UV})}$ achieve parity at $z \sim$ 0.4 , after which $\rho_{\star(\mathrm{rad})}$ continues to rise until $z \sim 0.7$ (cf. Cowie et al. 2004) when incompleteness seriously impacts the sample. Although it is tempting to speculate that the rise in $\rho_{\star(\mathrm{rad})}$ results from the increasing dominance of dust-obscured IR-luminous galaxies, we must recall our sample's origins. We are witnessing an increasing SFR per $U V$-selected galaxy, partly because at $z \sim 1$ we are probing the most UV-luminous galaxies; we are also witnessing an increase in $\rho_{\star(\mathrm{rad})}$ despite the increasing incompleteness. Adding $\mathrm{SFR}_{\mathrm{UV}}$ and $\mathrm{SFR}_{\mathrm{rad}}, \rho_{\star}$ due to UVselected galaxies increases as at least $(1+z)^{2.2}$ between $z=$ 0 and 1 (cf. Schiminovich et al. 2005).

The mismatch between the absolute and relative rates of SF derived using UV- and radio-based indicators is worrying, particularly the difference between local estimates of $\rho_{\star}$. The local $\rho_{\star(\mathrm{UV})}$ matches the $\rho_{\star}$ compilation presented by Hopkins $\&$ Beacom (2006), which implies that the UV-selected sample accounts for most of the SF in the local universe, yet the UV sample at $0.0<$ $z<0.2$ accounts for $\ll 1 \%$ of the total cataloged $S_{1.4 \mathrm{GHz}}$ in the region of common areal coverage. If the fraction of $S_{1.4 \mathrm{GHz}}$ due 
TABLE 1

$S_{1.4 \mathrm{GHz}}$ and SFRs Determined by Pixel Stacking at the Positions of Galaxies in the Samples (See $§ 4$ )

\begin{tabular}{|c|c|c|c|}
\hline Nos. ${ }^{a}$ & Selection Criteria & $S_{1.4 \mathrm{GHz}}$ & $\mathrm{SFR}^{\mathrm{b}}$ \\
\hline \multicolumn{4}{|c|}{ UV-selected galaxies, $R_{\mathrm{AB}}<24.1 \mathrm{mag}$} \\
\hline $136-0-0 \ldots$ & $z=0.0-0.2$ & $4.1 \pm 1.1$ & 0.12 \\
\hline $678-9-0 \ldots$ & $z=0.2-0.4$ & $11.2 \pm 0.5$ & 3.6 \\
\hline $612-12-1 \ldots \ldots \ldots$ & $z=0.4-0.6$ & $8.4 \pm 0.5$ & 8.9 \\
\hline $1055-20-2 \ldots \ldots \ldots$ & $z=0.6-0.8$ & $8.8 \pm 0.4$ & 21 \\
\hline $667-7-0 \ldots \ldots \ldots$ & $z=0.8-1.0$ & $8.5 \pm 0.5$ & 36 \\
\hline $441-7-5 \ldots \ldots \ldots$ & $z=1.0-1.2$ & $6.8 \pm 0.6$ & 47 \\
\hline $276-3-2 \ldots \ldots$ & $z=1.2-1.4$ & $5.7 \pm 0.8$ & 59 \\
\hline $30-1-1 \ldots \ldots \ldots \ldots$ & $z=1.4-1.6$ & $4.5 \pm 2.4$ & 65 \\
\hline \multicolumn{4}{|c|}{ EROs, $K_{s}<20.5 \mathrm{mag}, R-K_{s}>5.3 \mathrm{mag}$} \\
\hline $216-14-7 \ldots$ & $R-K_{s}=5.3-5.6$ & $13.6 \pm 0.8$ & 126 \\
\hline $170-15-8 \ldots$ & $R-K_{\mathrm{s}}>5.6$ & $11.9 \pm 0.9$ & 111 \\
\hline 35-7-4 „.......... & $K_{s}=17-18$ & $20.6 \pm 2.2$ & 194 \\
\hline $187-19-10 \ldots \ldots$ & $K_{s}^{s}=18-19$ & $16.9 \pm 0.9$ & 159 \\
\hline 140-3-1 $\ldots \ldots \ldots \ldots$ & $K_{s}=19-20$ & $6.3 \pm 1.0$ & 59 \\
\hline $19-0-0 \quad \ldots \ldots \ldots \ldots$ & $K_{s}^{s}=20-21$ & $12.9 \pm 2.8$ & 121 \\
\hline \multicolumn{4}{|c|}{ EROs, $R_{\mathrm{AB}}-3.6>4 \mathrm{mag}$} \\
\hline $1027-53-17$. & $R_{\mathrm{AB}}-3.6=4.0-4.5$ & $11.8 \pm 0.4$ & 81 \\
\hline $720-29-4 \ldots$. & $R_{\mathrm{AB}}-3.6=4.5-5.0$ & $10.6 \pm 0.4$ & 73 \\
\hline $437-29-8 \ldots$ & $R_{\mathrm{AB}}-3.6=5.0-5.5$ & $14.4 \pm 0.6$ & 100 \\
\hline $137-11-2 \ldots$ & $R_{\mathrm{AB}}-3.6=5.5-6.0$ & $16.7 \pm 1.0$ & 115 \\
\hline $42-8-1 \ldots \ldots$ & $R_{\mathrm{AB}}-3.6>6.0$ & $27.7 \pm 1.8$ & 192 \\
\hline $411-62-15$ & $3.6 \mu \mathrm{m}=19-20$ & $25.1 \pm 0.6$ & 174 \\
\hline $1032-48-9$. & $3.6 \mu \mathrm{m}=20-21$ & $13.1 \pm 0.4$ & 90 \\
\hline $742-10-1 \ldots \ldots \ldots$ & $3.6 \mu \mathrm{m}=21-22$ & $6.4 \pm 0.4$ & 44 \\
\hline $154-3-2 \ldots \ldots \ldots$ & $3.6 \mu \mathrm{m}>22$ & $4.6 \pm 0.9$ & 32 \\
\hline \multicolumn{4}{|c|}{$24 \mu \mathrm{m}$ galaxies, $S_{4.5}>S_{3.6}$} \\
\hline $634-65-291 \ldots \ldots$ & $S_{24}=0.15-0.3 \mathrm{mJy}$ & 23.5 & 1400 \\
\hline $196-61-89 \quad \ldots \ldots \ldots$ & $S_{24}=0.3-0.6 \mathrm{mJy}$ & 41.6 & 2480 \\
\hline $67-34-21 \ldots \ldots \ldots$ & $S_{24}=0.6-1.2 \mathrm{mJy}$ & 67.2 & 4010 \\
\hline $29-19-2 \ldots$ & $S_{24}>1.2 \mathrm{mJy}$ & 103 & 6120 \\
\hline $173-46-86 \ldots \ldots$ & $R_{\mathrm{AB}}<23$ & 38.0 & 2260 \\
\hline $154-39-67 \ldots \ldots$ & $R_{\mathrm{AB}}=23-24$ & 32.7 & 1950 \\
\hline $222-43-87 \ldots \ldots$ & $R_{\mathrm{AB}}=24-25$ & 27.5 & 1640 \\
\hline $151-26-69 \quad \ldots \ldots$. & $R_{\mathrm{AB}}^{\mathrm{AB}}=25-26$ & 29.0 & 1730 \\
\hline $92-7-41 \ldots \ldots \ldots$ & $R_{\mathrm{AB}}>26$ & 21.7 & 1290 \\
\hline \multicolumn{4}{|c|}{ DRGs, $J-K_{s}>2.3 \mathrm{mag}$} \\
\hline $80-2-1$ & $K_{s}<20.5$ & $10.1 \pm 1.3$ & 150 \\
\hline \multicolumn{4}{|c|}{ LBGs } \\
\hline $107-0-28 \ldots \ldots \ldots$ & $\cdots$ & $2.0 \pm 2.3$ & $<500$ \\
\hline \multicolumn{4}{|c|}{ ILLBGs } \\
\hline $6-1-4 \ldots \ldots \ldots \ldots$ & $S_{24}>0.06 \mathrm{mJy}$ & $44.2 \pm 11.5$ & 2120 \\
\hline
\end{tabular}

to obscured SF at $0.0<z<0.2$ exceeds $1 \%, \rho_{\star}$ would then be at the upper envelope of commonly accepted values.

Moving to slightly higher redshifts, we take two catalogs of EROs. One uses the conventional color cut $R-K_{s}>5.3$ with $K_{s}<20.5$ and DEEP2 redshifts, $1.0<z<1.5$ (C. J. Conselice et al. 2007, in preparation). It contains 382 objects in low-noise areas of our radio image. The second (Wilson et al. 2007) uses $R_{\mathrm{AB}}-3.6 \mu \mathrm{m}>4$ to select essentially the same class of objects, but the larger area covered at $3.6 \mu \mathrm{m}$ (and no requirement for redshifts) yields 2363 objects in $0.26 \mathrm{deg}^{2}$ of AEGIS20. We assumed $z=1.1$ to excise radio-loud AGNs from this sample.

Both ERO samples are well detected at $1.4 \mathrm{GHz}$, as shown in Figure 2. In the spectroscopic sample, the mean $S_{1.4 \mathrm{GHz}}$ does not vary significantly as a function of color, although it is a

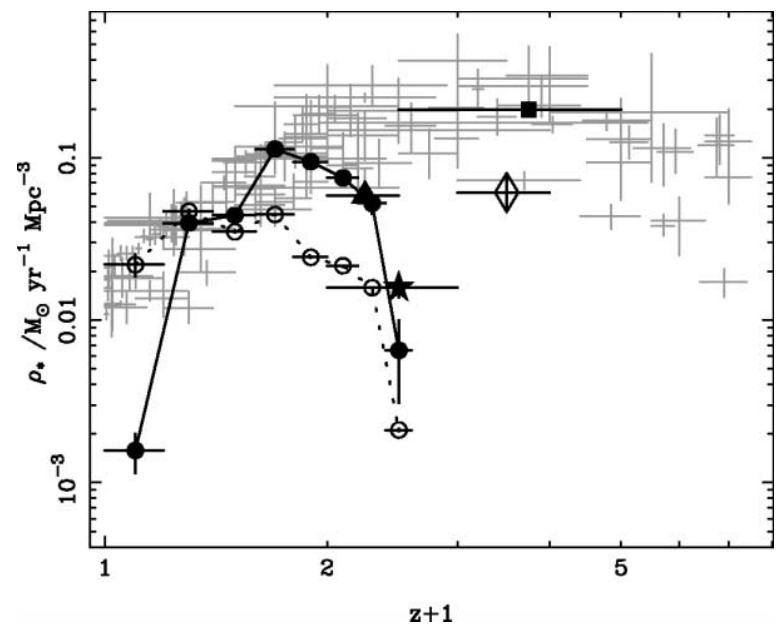

FIG. 1.-Radio-based estimates of SFR density $\left(\rho_{\star}\right)$ for a number of independent and overlapping galaxy populations selected via magnitude and color criteria: UV-selected galaxies (open and filled circles for UV-and radio-based SFRs, joined by dotted and solid lines), $R-K_{s}>5.3$ EROs (filled triangle), DRGs (filled star), ILLBGs (open diamond), and $24 \mu \mathrm{m}$-selected galaxies (filled square). These are conservative estimates-no attempt has been made to correct for accessible volume; contamination by radio-loud AGNs is possible at $z \gg 1(\S 5)$. The upper envelope of points thus traces the minimum $\rho_{\star}$ as a function of redshift, as demonstrated by the compilation of $\rho_{\star}$ data from Hopkins \& Beacom (2006), plotted faintly here. [See the electronic edition of the Journal for a color version of this figure.]

function of $K_{s}$ consistent with the findings of Smail et al. (2002). The median $L_{1.4 \mathrm{GHz}}$ is $8.1 \times 10^{22} \mathrm{~W} \mathrm{~Hz}^{-1}$, and the median SFR per ERO is $92 \pm 7 M_{\odot} \mathrm{yr}^{-1}$. In the sample volume set by the redshift limits and survey area, this equates to $\rho_{\star}=0.07 M_{\odot} \mathrm{yr}^{-1} \mathrm{Mpc}^{-3}$.

The larger ERO sample reveals a weak trend for $S_{1.4 \mathrm{GHz}}$ to increase with redness; this is confirmed by the increasing detection rate for individual objects (Table 1). The mean $S_{1.4 \mathrm{GHz}}$ also declines as $S_{3.6 \mu \mathrm{m}}$ decreases. We expect $S_{3.6 \mu \mathrm{m}}$ to trace stellar mass and distance, and the factor of $\sim 5.5$ decrease in $S_{1.4 \mathrm{GHz}}$ for a for a factor of $\sim 15$ decrease in $S_{3.6 \mu \mathrm{m}}$ suggests an increasing SFR per unit stellar mass as redshift increases. The overall $\rho_{\star}$ for this sample is consistent with that of the spectroscopic sample, as expected given the limited number of spectroscopic redshifts and the significant sample overlap.

J.-S. Huang et al. (2007, in preparation) present a catalog selected at $S_{24 \mu \mathrm{m}}>150 \mu \mathrm{Jy}$, with $S_{4.5 \mu \mathrm{m}}>S_{3.6 \mu \mathrm{m}}$, aiming to select galaxies and AGNs at $z>1.5$. Almost $10^{3}$ objects lie in low-noise regions of our radio mosaic, overlapping AEGIS20 by $0.26 \mathrm{deg}^{2}$. The individual radio detection rate is a strong function of $S_{24 \mu \mathrm{m}}$, rising from $30 \%$ to $70 \%$ between $S_{24 \mu \mathrm{m}}=0.15$ and $>1.2 \mathrm{mJy}$. The median $S_{1.4 \mathrm{GHz}}$ (Table 1) is fairly insensitive to $R_{\mathrm{AB}}$, varying by a factor of $<2$ over $>3 \mathrm{mag}$. Over $40 \%$ of the $24 \mu \mathrm{m}$-selected galaxies have $L_{1.4 \mathrm{GHz}}>10^{24} \mathrm{~W} \mathrm{~Hz}^{-1}$ when assuming $z=2.75$. At this redshift, all $\mathrm{S} / \mathrm{N} \geq 3$ measurements imply radio-loud AGNs, and it is difficult to estimate the SFR: $\rho_{\star}$ is likely to be high but so is the level of accretion-related contamination. The median $S_{1.4 \mathrm{GH}}, 28 \mu \mathrm{Jy}$, translates into $\rho_{\star}=0.20 M_{\odot} \mathrm{yr}^{-1} \mathrm{Mpc}^{-3}$ for $z=$ $1.5-4$. We would be unsurprised if this is in error by a factor of 2 ; regardless, this is an important star-forming population.

We now move on to yet more distant populations, this time to a sample of DRGs selected at $K_{s}<20.5$ (Vega) with $J-$ $K_{s}>2.3$ (Conselice et al. 2007), 108 of which lie within the $0.11 \mathrm{deg}^{2}$ of common coverage with AEGIS20. Although expected to lie at $1.9<z<3.5$ (Franx et al. 2003), Conselice et al. find that $64 \%$ lie at $1<z<2$. Galaxies with $z<1$, evident 


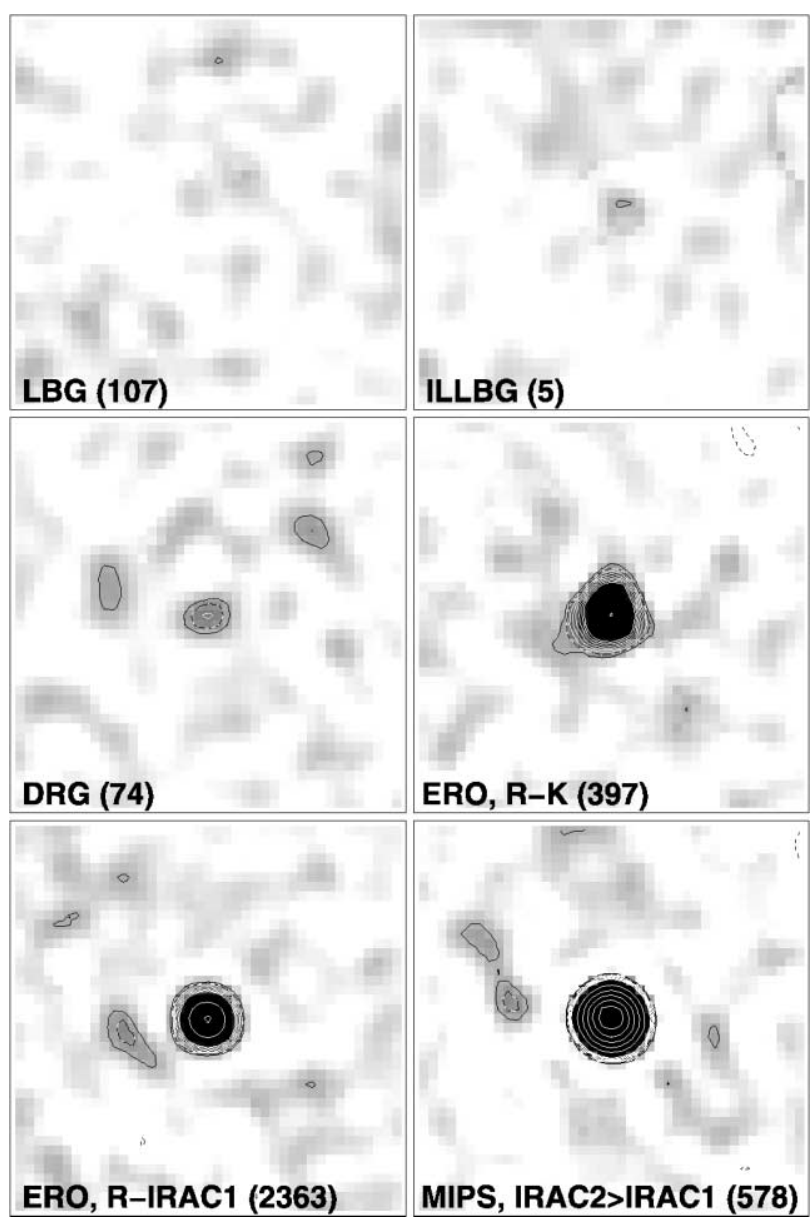

FIG. 2.-Stacked S/N images $\left(33^{\prime \prime} \times 33^{\prime \prime}\right)$ at the positions of the galaxies described in $\S 4$ : conventional and IR-luminous LBGs (top panels), DRGs and $R-K_{s}>5.3$ EROs (middle panels), and IRAC-selected EROs and MIPSselected galaxies with $S_{4.5 \mu \mathrm{m}}>S_{3.6 \mu \mathrm{m}}$ (bottom panels). Areas of high noise and galaxies detected individually ( $\geq 5 \sigma)$ were excluded. Contours are plotted at $\mathrm{S} / \mathrm{N}$ levels of $-3,3,4, \ldots, 10,20, \ldots, 100$, and the gray scale is identical in each case. The number of stacked sources from each population is shown in parentheses. The detection of the IRAC-selected ERO population is so significant that we see secondary structure: the image resembles the dirty beam since none of the individually undetected sources in the ensemble that makes up the stacked image have been CLEANed.

via DEEP2, have been removed from the sample used here. One of the radio-emitting DRGs has $L_{1.4 \mathrm{GHz}}$ consistent with radioloud AGNs (or, as noted earlier, a hyperluminous starburst). The mean $S_{1.4 \mathrm{GHz}}$ for the DRGs was $10.1 \pm 1.3 \mu \mathrm{Jy}$-faint emission can be seen in the stacked S/N image (Fig. 2). At $z=1.5$, this corresponds to $L_{1.4 \mathrm{GHz}}=(1.2 \pm 0.2) \times 10^{23} \mathrm{~W} \mathrm{~Hz}^{-1}$ and a mean SFR (per DRG) of $150 \pm 19 M_{\odot} \mathrm{yr}^{-1}$. Knudsen et al. (2005) found $190 \pm 50 M_{\odot} \mathrm{yr}^{-1}$ using submillimeter data for a sample of 30 DRGs (adapting to the cosmology and IMF used here), having assumed significantly larger distances. The observed radio emission from $K_{s}<20.5$ DRGs equates to $\rho_{\star}=$ $0.02 M_{\odot} \mathrm{yr}^{-1} \mathrm{Mpc}^{-3}$ at $z=1-2$.

The LBGs of Steidel et al. (2003) lie in a noisy region of the radio mosaic. Of the 334 cataloged LBGs, after correction for the astrometric offset in that catalog $\left(\Delta \alpha=+0.8^{\prime \prime}, \Delta \delta=+2.6^{\prime \prime}\right), 107$ lie within low-noise regions of the radio mosaic; their mean $S_{1.4 \mathrm{GHz}}$ was $2.0 \pm 2.3 \mu \mathrm{Jy}$ (median, $-0.6 \mu \mathrm{Jy}$ ), consistent with an average SFR of $<500 M_{\odot} \mathrm{yr}^{-1}(3 \sigma$, for $z=3$ ). Restricting the catalog to the 53 LBGs detected at $8 \mu \mathrm{m}$ with IRAC did not change the situation significantly (cf. Rigopoulou et al. 2006).

Finally, Huang et al. (2005) describe a population of IRluminous LBGs (ILLBGs) detected at $S_{24 \mu \mathrm{m}}>60 \mu \mathrm{Jy}$. Only six of their 13 objects lie within our radio mosaic. Their median $S_{1.4 \mathrm{GHz}}$ is $44.2 \mu \mathrm{Jy}$, including the one significant detection: Westphal MD99 at $\sim 1 \mathrm{mJy}$. This provides tentative support for the assertion that ILLBGs share the high SFRs of submillimeter galaxies, although this is a very small sample in a particularly noisy region of the radio mosaic, and accretion-related contamination is possible. If, as Huang et al. suggest, ILLBGs lie at $2<z<3$ (like submillimeter galaxies; Chapman et al. 2005), then their $\rho_{\star}$ is similar to that of the $24 \mu \mathrm{m}$-selected galaxies with which they will overlap significantly (Fig. 1).

\section{ON RADIO DATA AS A PROBE OF GLOBAL SF HISTORY}

Figure 1 shows $\rho_{\star}$ for the galaxy populations explored in $\S 4$. The upper envelope of points traces the minimum $\rho_{\star}$ as a function of redshift and appears to increase by at least a factor of 5 from $z=0$ to 1 , a now-familiar pattern (Lilly et al. 1996), although this work has led us to question the reliability of many SFR and $\rho_{\star}$ estimates.

Radio-based SFR estimates become increasingly prone to contamination by radio-loud AGNs at $z \gg 1$. Unfortunately, a consequence of removing this via a limit on $L_{1.4 \mathrm{GHz}}$ is the rejection of luminous star-forming galaxies obeying the far-IR/radio correlation; adopting a median $S_{1.4 \mathrm{GHz}}$ is unlikely to be better. In addition, some redshift-limited galaxy populations defined by color appear less well defined than first claimed (Conselice et al. 2007), limiting our ability to judge the volume probed. These effects lead to large uncertainties, so while it is clear that stacking radio data is useful, accurate SFRs for distant galaxies require precise redshifts together with deep, multifrequency, high-resolution radio data $\left(\ll 1^{\prime \prime}, \sigma_{0.6 \mathrm{GHz}} \sim \sigma_{1.4 \mathrm{GHz}} \lesssim 1 \mu \mathrm{Jy}\right)$. These will facilitate identification and removal of accretion-related emission via radio luminosity, spectral index, brightness temperature, and morphology.

\section{REFERENCES}

Arnouts, S., et al. 2005, ApJ, 619, L43

Bell, E. F. 2003, ApJ, 586, 794

Biggs, A. D., \& Ivison, R. J. 2006, MNRAS, 371, 963

Chapman, S. C., Blain, A. W., Smail, I., \& Ivison, R. J. 2005, ApJ, 622, 772

Condon, J. J. 1992, ARA\&A, 30, 575

Conselice, C. J., et al. 2007, ApJ, 660, L55

Cowie, L. L., Barger, A. J., Fomalont, E. B., \& Capak, P. 2004, ApJ, 603, L69

Davis, M., et al. 2007, ApJ, 660, L1

Dole, H., et al. 2006, A\&A, 451, 417

Fanaroff, B. L., \& Riley, J. M. 1974, MNRAS, 167, 31P

Fixsen, D. J., Dwek, E., Mather, J. C., Bennett, C. L., \& Shafer, R. A. 1998, ApJ, 508, 123

Fomalont, E. B., Windhorst, R. A., Kristian, J. A., \& Kellerman, K. I. 1991, AJ, 102, 1258

Franx, M., et al. 2003, ApJ, 587, L79

Helou, G., Soifer, B. T., \& Rowan-Robinson, M. 1985, ApJ, 298, L7
Hopkins, A. M., \& Beacom, J. F. 2006, ApJ, 651, 142

Huang, J.-S., et al. 2005, ApJ, 634, 137

Knudsen, K. K., et al. 2005, ApJ, 632, L9

Kovács, A., Chapman, S. C., Dowell, C. D., Blain, A. W., Ivison, R. J., Smail, I., \& Phillips, T. G. 2006, ApJ, 650, 592

Lilly, S. J., Le Fèvre, O., Hammer, F., \& Crampton, D. 1996, ApJ, 460, L1

Rigopoulou, D., et al. 2006, ApJ, 648, 81

Salim, S., et al. 2005, ApJ, 619, L39

Schiminovich, D., et al. 2005, ApJ, 619, L47

Smail, I., Owen, F. N., Morrison, G. E., Keel, W. C., Ivison, R. J., \& Ledlow, M. J. 2002, ApJ, 581, 844

Spergel, D. N., et al. 2003, ApJS, 148, 175

Steidel, C. C., Adelberger, K. L., Shapley, A. E., Pettini, M., Dickinson, M., \& Giavalisco, M. 2003, ApJ, 592, 728

Wilson G., et al. 2007, ApJ, 660, L59 